\title{
Optimal Cutoff Scores for Dementia and Mild Cognitive Impairment in the Brazilian Version of the Montreal Cognitive Assessment among the Elderly
}

\author{
Tiago C.C. Pinto ${ }^{a}$ b Marília S.P. Santos ${ }^{a}$ Leonardo Machado ${ }^{a}$ \\ Tatiana M. Bulgacov ${ }^{a}$ Antônio L. Rodrigues-Junior ${ }^{a, c}$ Gabriela A. Silva ${ }^{a}$ \\ Maria Lúcia G. Costa ${ }^{a}$ d Rosana C.C. Ximenes ${ }^{a}$ Everton B. Sougey ${ }^{a}$ \\ a Postgraduate Program in Neuropsychiatry and Behavioral Science at the Universidade Federal \\ de Pernambuco (UFPE), Recife, Brazil; ${ }^{b}$ University Hospital Doctor Washington Antônio de Barros \\ of Universidade Federal do Vale do São Francisco (UNIVASF), Petrolina, Brazil; ' $B$ Busca Vida Clinical \\ Gerontology, Recife, Brazil; ${ }^{d}$ Post-graduate program in Gerontology at the Federal University of \\ Pernambuco, Recife, Brazil
}

\section{Keywords}

Mild cognitive impairment - Alzheimer's disease - Dementia - Screening for cognitive impairment · Elderly · Montreal Cognitive Assessment

\section{Abstract}

Objective: To propose cutoff scores for the Brazilian version of the Montreal Cognitive Assessment (MoCA-BR) stratified by education in order to detect mild cognitive impairment $(\mathrm{MCl})$ and mild Alzheimer's disease (AD) in the elderly. Method: A transversal study in health centers was performed on 159 elderly people with 4-12 years of education and 70 of their peers with over 12 years of schooling. The MoCA-BR cutoff scores for screening cognitive impairment were determined based on an ROC curve analysis. Results: The ROC curve analysis indicated that cutoff scores under 20 were good for screening elderly people with cognitive impairment with more than 12 years of education, and scores under 21 were good for screening those with 4-12 years of education. Conclusions: MoCA-BR scores under 21 points (after adding 1 point to the elderly with $\leq 12$ years of education) indicate a need to continue the diagnostic investigation with regular follow-ups. 


\section{Introduction}

The distinction between cognitive alterations compatible with normal aging and pathological processes in the early stages, such as mild cognitive impairment (MCI) and mild AD, is sometimes difficult [1]. The normal aging process is associated with declines in processing speed and certain memory, language, visuospatial, and executive function abilities [2]. For this distinction, cognitive tests, including the Brazilian version of the Montreal Cognitive Assessment (MoCA-BR), were used. The MoCA is an instrument developed by Nasreddine et al. [3] as an MCI screening test.

This test has been proven to be sensitive to $\mathrm{MCI}$ and to predict future cognitive decline in several cognitively impaired states, including AD [4]. It represents a practical and effective method for differentiating between the cognitive performance of elderly people with normal cognitive aging and those with MCI. It is also useful in differentiating between MCI and AD in mild to moderate stages. The time for its application is 10-15 min [3, 5], with a maximum score of 30 . The MoCA-BR was evaluated in a sample of 112 people over 65 years of age with at least 4 years of education, divided into groups according to their cognitive state [6].

Education is considered a criterion for the establishment of normative data for cognitive tests, and the impact the level of education has on cognitive performance has been well established in the literature [5, 7-15]. Furthermore, the author of the MoCA proposed adding 1 point to individuals with 12 years of education or less, aiming to correct the effect education has on MoCA performance [3].

Additionally, studies have determined different MoCA cutoff scores to discriminate individuals with MCI from cognitively healthy individuals, i.e., $<26[3,16,17],<23[18],<22[19$, $20],<21$ [13], and $<20[21,22]$. Some studies $[6,12,18,23-25]$ have revealed that the originally recommended cutoff score of 26 leads to a higher rate of false positives than that found in the original study of Nasreddine et al. [3]. A recent meta-analysis revealed that an MoCA cutoff score of 23 lowers the false-positive rate and shows an overall better diagnostic accuracy [26]. Therefore, the present study aims to propose MoCA-BR cutoff scores stratified by education in order to detect MCI and mild AD in the elderly.

\section{Materials and Methods}

\section{Study Design, Sample, Procedure, and Instruments}

This is an observational, transversal, analytical study conducted at 4 respected health care centers for the elderly in Recife, Brazil. The population in the study was composed of people aged 65 years and older, of both genders, with at least 4 years of education. The participants were already being followed up in some of the centers where the study was conducted. They spontaneously sought medical care due to their memory complaints. Patients were also sent from other units for evaluation of suspected cognitive impairment. Other participants were healthy people from the community who were willing to participate in this study.

The participants were divided into the following 3 groups: control (cognitively healthy people), $\mathrm{MCI}$ (patients with $\mathrm{MCI}$ ), and mild $\mathrm{AD}$ (people with an $\mathrm{AD}$ clinical dementia rating [CDR] of 1). All of the participants in this study were submitted to anamnesis ranging from medical history to habits, and the application of sociodemographic and clinical questionnaires containing general facts, i.e., age, gender, education, comorbidities, and medication. For evaluation of the functionality of the elderly, the Pfeffer Functional Activities Questionnaire [27] was applied by an experienced occupational therapist. For cognitive evaluation of the elderly in this study, a complete neuropsychological evaluation was performed by a 
neuropsychologist. To evaluate the stages of dementia, CDR, prepared by Hughes et al. [28] and updated by Morris [29], was used.

The inclusion and exclusion criteria for each group are described below.

- The inclusion criteria for the control group were: age $\geq 65$ years and education $\geq 4$ years.

- The exclusion criteria for the control group were: a diagnosis of dementia; a diagnosis of relevant neurological or psychiatric diseases or systematic uncontrolled chronic diseases that have an impact on cognition; a history of alcoholism or substance abuse; aphasia or a visual, hearing, or motor handicap; evidence of loss of autonomy or independence in daily activities; regular use of psychotropic drugs; and altered performance on neuropsychological tests.

- The inclusion criteria of the MCI group were: age $\geq 65$ years; education $\geq 4$ years; a subjective cognitive complaint, preferably confirmed by an informant; an objective cognitive deficit confirmed by a low performance under the level expected on neuropsychological tests; normal general cognitive functions; and intact or minimally impaired functional activities. The exclusion criterion for the MCI group was a diagnosis of dementia.

- The inclusion criteria for the $\mathrm{AD}$ group were: age $\geq 65$ years; education $\geq 4$ years; a clinical diagnosis of Alzheimer's disease (AD), supported by neuropsychological tests showing cognitive impairment and a functional evaluation showing a decrease in functionality; and mild stage dementia, evaluated through CDR, corresponding to a score equal to 1 using this tool. The exclusion criteria for the AD group were: a diagnosis of mixed dementia (AD associated with another type of dementia), and moderate or advanced stages of dementia evaluated by CDR.

Considering the inclusion and exclusion criteria in the medical interview, past and current medical histories of the elderly, and the performance of a complete neuropsychological evaluation performed by an experience professional, a team composed of a psychiatrist, a neuropsychologist, and a geriatric doctor classified, following a consensus meeting, the participants selected for the control group, the MCI group, and the AD CDR 1 group. The occupational therapist who applied the MoCA-BR did not participate in the consensus meeting for selection and classification of the elderly, in an effort to avoid the risk of bias by using the information on the MoCA-BR performance for the definition of a cognitive diagnosis. The MCI diagnosis was defined based on the criteria of Petersen [30]. In turn, the diagnosis of AD was based on the criteria of the National Institute of Neurological and Communicative Disorders and Stroke/Alzheimer's Disease and Related Disorders Association (NINCDS/ADRA) [31].

\section{Statistical Analysis}

For the statistical analysis, the Statistical Package for the Social Sciences (SPSS), version 21.0, was used. Application of the Kolmogorov-Smirnov and Shapiro-Wilk tests demonstrated an abnormal distribution of the MoCA-BR outcome score variable. Therefore, nonparametric tests were used to apply hypothesis tests. For comparison of performances on the MoCA-BR among the control, $\mathrm{MCI}$, and $\mathrm{AD}$ groups, a nonparametric Kruskal-Wallis test, with use of the Dunn-Bonferroni test for post hoc analysis, was applied.

To determine the accuracy of the MoCA-BR in discriminating between the control and $\mathrm{MCI}$, control and $\mathrm{AD}$, and $\mathrm{MCI}$ and $\mathrm{AD}, \mathrm{ROC}$ curves and determination of the area under the curve were used. Contingency tables $(2 \times 2)$ were used to calculate the number of individuals who were correctly classified regarding their cognitive state. The optimum cutoff scores stratified according to education range to detect cognitive impairment were established using the Youden index (calculated using the formula: sensitivity + specificity -1 ) and based on the percentage of those correctly classified. For rejection of the null hypothesis, a value of $p<0.05$ and a CI of $95 \%$ were considered as significant levels. 


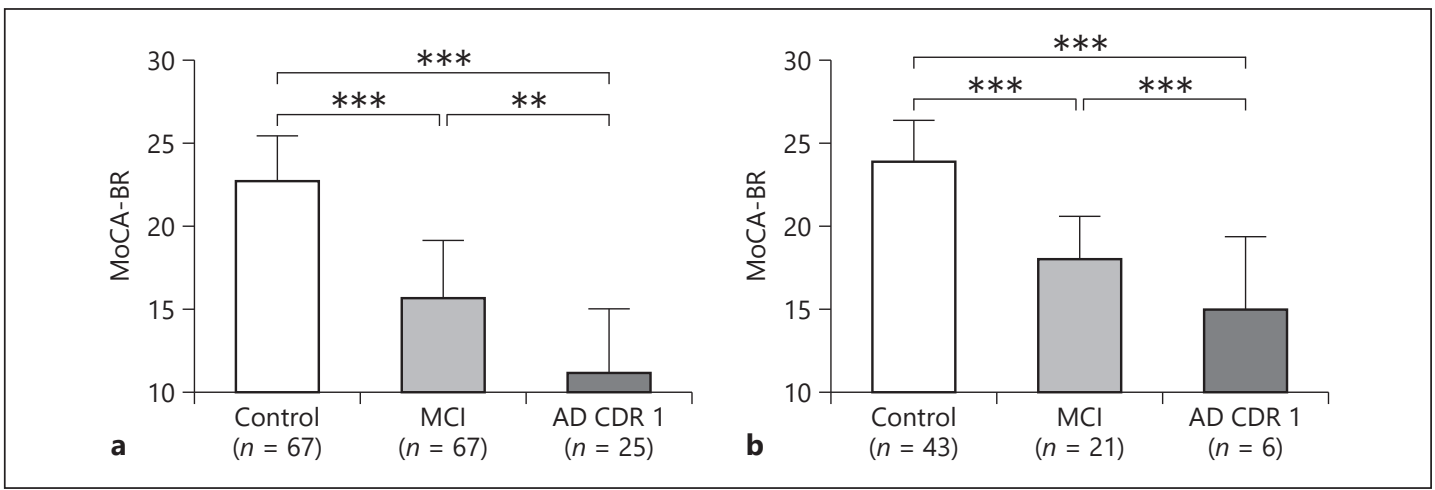

Fig. 1. Comparison between the performance of the cognitively healthy group (control), those with MCI, and those with mild AD (AD CDR 1 group) on the MoCA-BR in participants with 4-12 (a) and more than 12 years of education (b). There was a significant statistical difference between the scores of the 3 groups in both education ranges ( $p<0.001$; Kruskal-Wallis test). ${ }^{* *} p \leq 0.01 ;{ }^{* * *} p<0.001$, obtained through post hoc analysis with the Dunn-Bonferroni test.

\section{Results}

From a total of 159 elderly people with 4-12 years of education, 25 (15.8\%) received an AD CDR 1 diagnosis, 67 (42.1\%) were classified as having MCI, and 67 (42.1\%) were classified as being cognitively healthy. One hundred twenty-six (79.2\%) of the participants were female, and the average age was $74.62 \pm 6.18$ years. In turn, the average age of the 70 people with more than 12 years of education was $72.13 \pm 5.76$ years, and they were predominantly female (70.0\%). There was no statistical difference between the diagnostic groups of both education ranges regarding gender or age $(p<0.05)$. There was a difference in cognitive performance between those with 4-12 years of education and those with over 12 years of education ( $p<$ 0.001 , Mann-Whitney test).

When comparing the performances of the control, MCI, and AD CDR 1 groups on the MoCA-BR, a significant statistical difference was found between the scores of the 3 groups $(p<0.001)$ in both education ranges (Fig. 1). The results of the evaluation of the validity of the MoCA-BR to discriminate among ROC curves with stratification by education range is presented in Figure 2. The MoCA-BR areas under the curve stratified by range of education are presented in Table 1.

The cutoff point designated by the MoCA-BR author is $25 / 26$, indicating that points lower than 26 are probably MCI or dementia cases. When using this cutoff point in the present study, even though an elevated sensitivity $(100.0 \%)$ was found, the specificity of the test was very low $(21.8 \%)$, with only $62.4 \%$ of the participants correctly classified. Table 2 presents the optimum cutoff scores stratified according to each education range to detect cognitive impairment.

In the cutoff scores presented in the present study, the percentage of people correctly classified regarding their cognitive state was very high, varying from 84.3 to $98.9 \%$. The sensitivity and specificity of the MoCA-BR were very expressive, varying from 83 to $96 \%$ and from 84 to $100 \%$, respectively, in all of the ideal cutoff scores. To screen the cases of mild AD, the ideal cutoff point was $17 / 18$, achieving over $95 \%$ hits regarding the cognitive state of the participants, showing excellent accuracy for the MoCA-BR in detecting cases of MCI as well as cases of mild AD. 


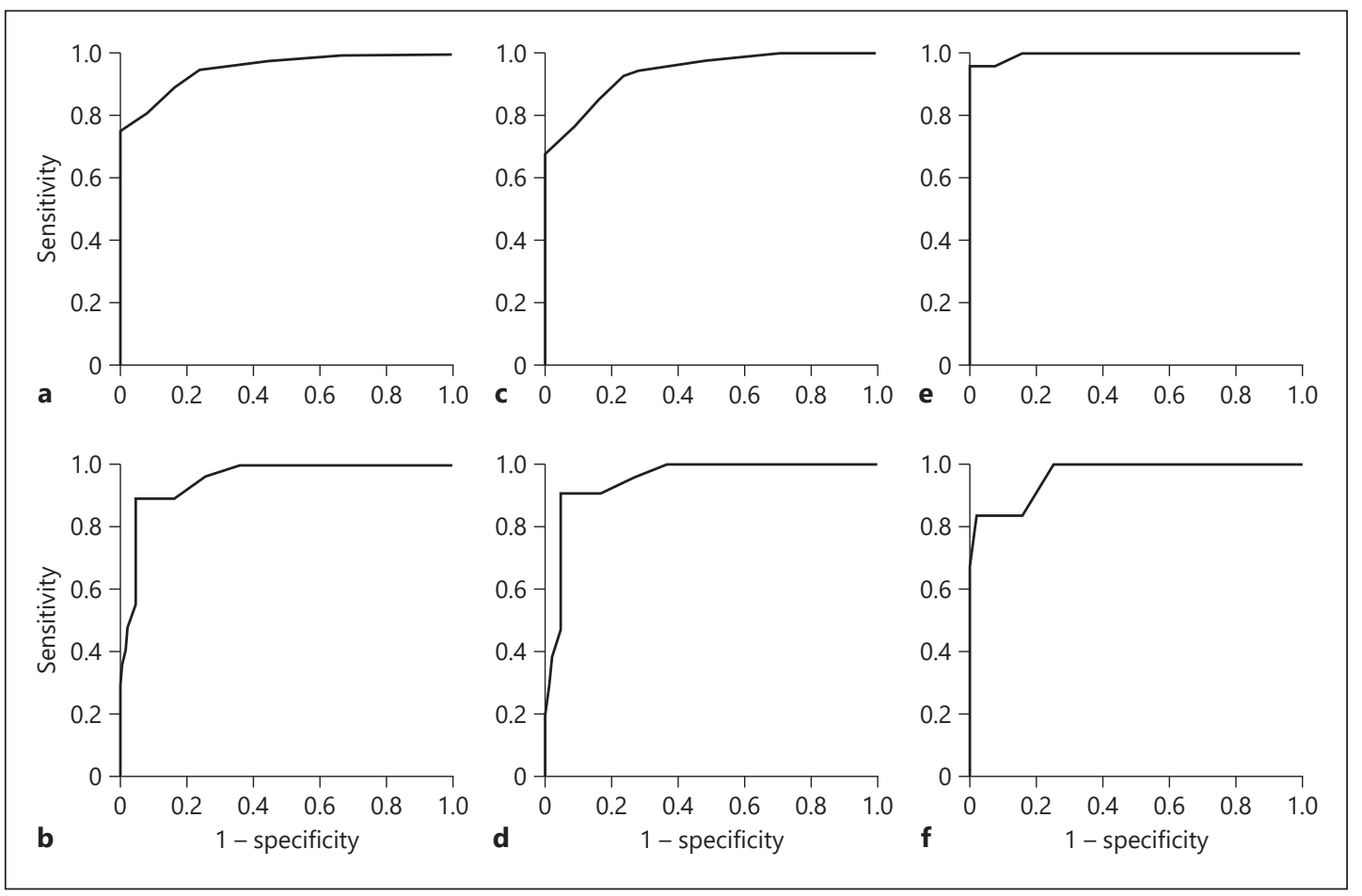

Fig. 2. ROC curves for the MoCA-BR to differentiate individuals with MCI and AD CDR 1 from cognitively normal controls according to education range. a Control group vs. group with cognitive impairment (MCI and AD CDR 1) in elderly subjects with 4-12 years of education. b Control group vs. group with cognitive impairment (MCI and AD CDR 1) (more than 12 years of education). c Control group vs. MCI group (4-12 years of education). $\mathbf{d}$ Control group vs. MCI group (more than 12 years of education). e Control group vs. AD CDR 1 group (4-12 years of education). $\mathbf{f}$ Control group vs. AD CDR 1 group (more than 12 years of education).

Table 1. Accuracy of the MoCA-BR in discriminating between cognitively healthy individuals and those with cognitive impairment, stratified by education

\begin{tabular}{|c|c|c|c|c|}
\hline & AUC & SE & $95 \% \mathrm{CI}$ & $\begin{array}{l}p \text { value } \\
(\text { area }=0.5)^{\mathrm{a}}\end{array}$ \\
\hline \multicolumn{5}{|l|}{ Control vs. MCI and AD CDR 1} \\
\hline 4-12 years of education & 0.952 & 0.0146 & $0.907-0.980$ & $<0.001$ \\
\hline More than 12 years of education & 0.953 & 0.0232 & $0.873-0.989$ & $<0.001$ \\
\hline \multicolumn{5}{|l|}{ Control vs. MCI } \\
\hline 4-12 years of education & 0.936 & 0.0193 & $0.881-0.971$ & $<0.001$ \\
\hline More than 12 years of education & 0.950 & 0.0266 & $0.864-0.989$ & $<0.001$ \\
\hline \multicolumn{5}{|l|}{ Control vs. AD CDR 1} \\
\hline 4-12 years of education & 0.995 & 0.0050 & $0.952-1.000$ & $<0.001$ \\
\hline More than 12 years of education & 0.963 & 0.0361 & $0.866-0.996$ & $<0.001$ \\
\hline
\end{tabular}


Pinto et al.: Optimal Cutoff Scores of the MoCA-BR among the Elderly

Table 2. Cutoff scores of the MoCA-BR, according years of education

\begin{tabular}{|c|c|c|c|c|c|c|c|}
\hline & $\begin{array}{l}\text { Cutoff } \\
\text { point }\end{array}$ & $\begin{array}{l}\text { Sensitivity, } \\
\%\end{array}$ & $\begin{array}{l}\text { Specificity, } \\
\%\end{array}$ & $\begin{array}{l}\text { False } \\
\text { negative, \% }\end{array}$ & $\begin{array}{l}\text { False } \\
\text { positive, \% }\end{array}$ & $\begin{array}{l}\text { Youden } \\
\text { index }\end{array}$ & $\begin{array}{l}\text { Correctly } \\
\text { classified } \\
\text { cases, } \%\end{array}$ \\
\hline \multicolumn{8}{|l|}{ Control vs. MCI and AD CDR 1} \\
\hline 4-12 years of education & $19-20$ & 89.1 & 83.6 & 10.9 & 16.4 & 0.727 & 86.8 \\
\hline More than 12 years of education & $20-21$ & 88.9 & 95.3 & 11.1 & 4.7 & 0.842 & 92.9 \\
\hline \multicolumn{8}{|c|}{ Control vs. MCI } \\
\hline 4-12 years of education & $19-20$ & 85.1 & 83.6 & 14.9 & 16.4 & 0.687 & 84.3 \\
\hline More than 12 years of education & $20-21$ & 90.5 & 95.3 & 9.5 & 4.7 & 0.858 & 93.8 \\
\hline \multicolumn{8}{|l|}{ Control vs. AD CDR 1} \\
\hline 4-12 years of education & $17-18$ & 96.0 & 100.0 & 4.0 & 0.0 & 0.960 & 98.9 \\
\hline More than 12 years of education & $17-18$ & 83.3 & 97.7 & 16.7 & 2.3 & 0.810 & 95.9 \\
\hline
\end{tabular}

\section{Discussion}

In the present study, cutoff scores for the detection of MCI and mild AD stratified by education range were proposed. As observed in others cognitive tests, MoCA-BR performance was affected by education [5, 7-10]. Previous studies in other countries have reported similar effects in relation to education, with people with lower levels of education obtaining lower scores $[5,11,13,18,19,33,34]$. Due to the effect of education on the performance on the MoCA-BR, the cutoff scores were determined according to the education range.

To identify the cases of MCI, the MoCA-BR presented the cutoff of point less than 21 as ideal for people with over 12 years of education, and that of less than 20 for those with 4-12 years of education. Therefore, the cutoff point for those with more than 12 years of education was higher than for those with less education, according to the findings of other studies [35-37], highlighting the importance of considering the level of education when evaluating cognitive performance to achieve a greater accuracy in screening diagnosis. This study presented an elevated sensitivity and a low percentage of false negatives in the MoCA-BR in the screening of cognitive impairment. The cutoff scores proposed in the present study for the detection of MCI were higher than those for the detection of mild $A D$, since there is a lower cognitive decline in $\mathrm{MCI}$. In other words, the performance of elderly with $\mathrm{MCI}$ is superior to that of those with AD.

Therefore, to increase the sensitivity of the test, based on an ROC curve analysis and comparison of the parameters of accuracy, a stricter cutoff point was found which was represented, in the case of the MoCA-BR battery, by values higher than those of AD (i.e., $\leq 19$ and $\leq 20$ vs. $\leq 17$, respectively). Findings similar to these have been reported in other studies $[6$, $24,23,33,38]$. Because it is a more rigorous cognitive screening test, the MoCA-BR is a useful tool in groups where the ceiling effect is a problem.

The excellent accuracy of the MoCA-BR when using the cutoff scores proposed in the present study was in contrast with the low percentage of correct classifications when using the cutoff point proposed by the author of the MoCA (62.4\%) [3], which is compatible with the results found in a recent meta-analysis [26]. This shows the importance of adapting the cutoff point according to the population under study. Other studies that used other versions of the MoCA also proposed cutoff scores inferior to those proposed by Nasreddine et al. [3], from values similar to those in the present study $[22,38]$ to a cutoff point of $14 / 15$ in a study conducted in Spain with elderly people in the control and $\mathrm{AD}$ groups with an average education less than that of those in present study [39]. With the recommendation that 1 point be added to the MoCA-BR score obtained for participants with up to 12 years of education - similar to that recommended by the 
MoCA author [3] - the cutoff point suggested in the present study is $20 / 21$ to discriminate between the cognitively healthy individuals and those with mild cognition impairment. People with fewer than 21 points must be referred for follow-up and a clear diagnosis.

The strengths of the present study are the use of NINCDS/ADRA criteria [31] for diagnosing $\mathrm{AD}$, the MCI diagnosis based on the criteria of Petersen criteria [30], cutoff points stratified by education level, and complete characterization of the subject sample, employing not only standardized examinations and instruments but also neuropsychological testing. The designations of cognitively impaired and cognitively healthy were based on objective findings from neuropsychological testing. Additionally, this study is relevant due to the relative scarcity, even in the international literature and especially in the Brazilian literature, of studies proposing MoCA-BR cutoff scores stratified by education in order to detect MCI and mild AD. The use of a sample with a distribution close to that observed in the elderly population increases the equivalence with the target population.

The present study has limitations that should be addressed. Participants with less than 4 years of education were excluded. The MoCA presents items that are inadequate for individuals with little or no formal education (especially the items related to executive functions) $[38,40]$. The use of the MoCA in this group would lead to a loss of discriminating power between normal and pathological, with an increase in false positives and false negatives, as occurred in studies which included illiterate or poorly educated older people [13, 34]. Moreover, the original MoCA study was developed for cognitive screening of people with a minimum formal education of 4 years [3]. Therefore, application of the results of this study to elderly people who have less than 4 years of education is not recommended. Another limitation is that the participants were recruited from one city in Brazil. Brazil is a country with continental dimensions, with a considerable socieodemographic and cultural regional variety, which could influence the performance of the elderly on the MoCA-BR. Thus, the cutoff points of this study should not be generalized or extrapolated to populations with characteristics different from those of the population in question, especially regarding education. However, the participants were randomly selected in proportions that were consistent within the elderly Brazilian population. The discrepancy was thus minimized. We suggest further studies recruiting participants from all over Brazil.

Concluding, the results of the new study indicate that cutoff scores of less than 21 and less than 20 for elderly people with 12 and 4-12 years of education, respectively, present excellent accuracy for diagnostic screening of cognitive impairment. Scores of under 21 points on the MoCA-BR - after adding 1 point to elderly individuals with $\leq 12$ years of education indicate a need for further diagnostic investigation and regular follow-up. The cutoff points presented can be used to inform future work using the MoCA-BR to screen for MCI and AD in older Brazilian people. Future studies could focus on early detection and treatment of cognitive dysfunctions in clinical practice.

\section{Acknowledgement}

The authors have no financial or nonfinancial affiliations with any organizations in relation to the subject matter or material discussed in this paper.

\section{Statement of Ethics}

The subjects gave their informed consent and the study protocol was approved by the institute's committee on human research. 
Pinto et al.: Optimal Cutoff Scores of the MoCA-BR among the Elderly

\section{Disclosure Statement}

The authors declare no potential conflict of interests with respect to the research, authorship, and/or publication of this article.

\section{References}

1 Petersen RC. Clinical practice. Mild cognitive impairment. N Engl J Med. 2011 Jun;364(23):2227-34.

2 Harada CN, Natelson Love MC, Triebel KL. Normal cognitive aging. Clin Geriatr Med. 2013 Nov;29(4):737-52.

3 Nasreddine ZS, Phillips NA, Bédirian V, Charbonneau S, Whitehead V, Collin I, et al. The Montreal Cognitive Assessment, MoCA: a brief screening tool for mild cognitive impairment. J Am Geriatr Soc. 2005 Apr;53(4): 695-9.

4 Davis DH, Creavin ST, Yip JL, Noel-Storr AH, Brayne CE, Cullum S: Montreal Cognitive Assessment for the diagnosis of Alzheimer's disease and other dementias. Cochrane Database Syst Rev. 2015 Oct; 10:CD010775.

5 Freitas S, Simões MR, Alves L, Santana I. Montreal Cognitive Assessment: influence of sociodemographic and health variables. Arch Clin Neuropsychol. 2012 Mar;27(2):165-75.

6 Memória CM, Yassuda MS, Nakano EY, Forlenza OV. Brief screening for mild cognitive impairment: validation of the Brazilian version of the Montreal cognitive assessment. Int J Geriatr Psychiatry. 2013 Jan;28(1):34-40.

7 Huppert FA, Brayne C, Gill C, Paykel ES, Beardsall L. CAMCOG-a concise neuropsychological test to assist dementia diagnosis: socio-demographic determinants in an elderly population sample. Br J Clin Psychol. 1995 Nov;34(Pt 4):529-41.

8 Mathuranath PS, Cherian JP, Mathew R, George A, Alexander A, Sarma SP. Mini mental state examination and the Addenbrooke's cognitive examination: effect of education and norms for a multicultural population. Neurol India. 2007 Apr-Jun;55(2):106-10.

9 Moraes C, Pinto JA Jr, Lopes MA, Litvoc J, Bottino CM. Impact of sociodemographic and health variables on mini-mental state examination in a community-based sample of older people. Eur Arch Psychiatry Clin Neurosci. 2010 Oct;260(7):535-42.

10 Ribeiro PC, Oliveira BH, Cupertino AP, Neri AL, Yassuda MS. Desempenho de idosos na bateria cognitiva CERAD: relações com variáveis sociodemográficas e saúde percebida. Psicol Reflex Crit. 2010;23(1):102-9.

11 Lu J, Li D, Li F, Zhou A, Wang F, Zuo X, et al. Montreal cognitive assessment in detecting cognitive impairment in Chinese elderly individuals: a population-based study. J Geriatr Psychiatry Neurol. 2011 Dec;24(4):184-90.

12 Rossetti HC, Lacritz LH, Cullum CM, Weiner MF. Normative data for the Montreal Cognitive Assessment (MoCA) in a population-based sample. Neurology. 2011 Sep; 77(13):1272-5.

13 Zhou S, Zhu J, Zhang N, Wang B, Li T, Lv X, et al. The influence of education on Chinese version of Montreal cognitive assessment in detecting amnesic mild cognitive impairment among older people in a Beijing rural community. Sci World J. 2014;2014:689456.

14 Larouche E, Tremblay MP, Potvin O, Laforest S, Bergeron D, Laforce R, et al. Normative data for the Montreal Cognitive Assessment in middle-aged and elderly Quebec-French people. Arch Clin Neuropsychol. 2016 Sep; 31(7):819-26.

15 Pinto TC, Machado L, Bulgacov TM, Rodrigues-Júnior AL, Costa ML, Ximenes RC, et al. Influence of age and education on the performance of elderly in the Brazilian version of the Montreal Cognitive Assessment battery. Dement Geriatr Cogn Disord. 2018;45(5-6):290-9.

16 Fujiwara Y, Suzuki H, Yasunaga M, Sugiyama M, Ijuin M, Sakuma N, et al. Brief screening tool for mild cognitive impairment in older Japanese: validation of the Japanese version of the Montreal Cognitive Assessment. Geriatr Gerontol Int. 2010 Jul;10(3):225-32.

17 Lifshitz M, Dwolatzky T, Press Y. Validation of the Hebrew version of the MoCA test as a screening instrument for the early detection of mild cognitive impairment in elderly individuals. J Geriatr Psychiatry Neurol. 2012 Sep;25(3):155-61.

18 Lee JY, Cho SJ, Na DL, Kim SK, Youn JH, Kwon M, et al.; Dong Woo Lee; Hong Jin Jeon; You Ra Lee; Maeng Je Cho. Brief screening for mild cognitive impairment in elderly outpatient clinic: validation of the Korean version of the Montreal Cognitive Assessment. J Geriatr Psychiatry Neurol. 2008 Jun;21(2):104-10.

$19 \mathrm{Yu} \mathrm{J,} \mathrm{Li} \mathrm{J,} \mathrm{Huang} \mathrm{X.} \mathrm{The} \mathrm{Beijing} \mathrm{version} \mathrm{of} \mathrm{the} \mathrm{Montreal} \mathrm{Cognitive} \mathrm{Assessment} \mathrm{as} \mathrm{a} \mathrm{brief} \mathrm{screening} \mathrm{tool} \mathrm{for} \mathrm{mild}$ cognitive impairment: a community-based study. BMC Psychiatry. 2012 Sep;12(1):156.

20 Janelidze M, Mikeladze N, Bochorishvili N, Dzagnidze A, Kapianidze M, Mikava N, et al. Validity of the Georgian Montreal Cognitive Assessment for the Screening of Mild Cognitive Impairment and Dementia. Am J Alzheimers Dis Other Demen. 2017 Feb;32(1):36-40.

21 Dong Y, Lee WY, Basri NA, Collinson SL, Merchant RA, Venketasubramanian N, et al. The Montreal Cognitive Assessment is superior to the Mini-Mental State Examination in detecting patients at higher risk of dementia. Int Psychogeriatr. 2012 Nov;24(11):1749-55.

22 Dong Y, Yean Lee W, Hilal S, Saini M, Wong TY, Chen CL, et al. Comparison of the Montreal Cognitive Assessment and the Mini-Mental State Examination in detecting multi-domain mild cognitive impairment in a Chinese subsample drawn from a population-based study. Int Psychogeriatr. 2013 Nov;25(11):1831-8. 
23 Tsai CF, Lee WJ, Wang SJ, Shia BC, Nasreddine Z, Fuh JL. Psychometrics of the Montreal Cognitive Assessment (MoCA) and its subscales: validation of the Taiwanese version of the MoCA and an item response theory analysis. Int Psychogeriatr. 2012 Apr;24(4):651-8.

24 Roalf DR, Moberg PJ, Xie SX, Wolk DA, Moelter ST, Arnold SE. Comparative accuracies of two common screening instruments for classification of Alzheimer's disease, mild cognitive impairment, and healthy aging. Alzheimers Dement. 2013 Sep;9(5):529-37.

25 Kaya Y, Aki OE, Can UA, Derle E, Kibaroğlu S, Barak A. Validation of Montreal Cognitive Assessment and discriminant power of Montreal Cognitive Assessment subtests in patients with mild cognitive impairment and Alzheimer dementia in Turkish population. J Geriatr Psychiatry Neurol. 2014 Jun;27(2):103-9.

26 Carson N, Leach L, Murphy KJ. A re-examination of Montreal Cognitive Assessment (MoCA) cutoff scores. Int J Geriatr Psychiatry. 2018 Feb;33(2):379-88.

27 Pfeffer RI, Kurosaki TT, Harrah CH Jr, Chance JM, Filos S. Measurement of functional activities in older adults in the community. J Gerontol. 1982 May;37(3):323-9.

28 Hughes CP, Berg L, Danziger WL, Coben LA, Martin RL. A new clinical scale for the staging of dementia. Br J Psychiatry. 1982 Jun;140(6):566-72.

29 Morris JC. The Clinical Dementia Rating (CDR): current version and scoring rules. Neurology. 1993 Nov; 43(11):2412-4.

30 Petersen RC. Mild cognitive impairment as a diagnostic entity. J Intern Med. 2004 Sep;256(3):183-94.

31 McKhann G, Drachman D, Folstein M, Katzman R, Price D, Stadlan EM. Clinical diagnosis of Alzheimer's disease: report of the NINCDS-ADRDA Work Group under the auspices of Department of Health and Human Services Task Force on Alzheimer's Disease. Neurology. 1984 Jul;34(7):939-44.

32 DeLong ER, DeLong DM, Clarke-Pearson DL. Comparing the areas under two or more correlated receiver operating characteristic curves: a nonparametric approach. Biometrics. 1988 Sep;44(3):837-45.

$33 \mathrm{Hu}$ JB, Zhou WH, Hu SH, Huang ML, Wei N, Qi HL, et al. Cross-cultural difference and validation of the Chinese version of Montreal Cognitive Assessment in older adults residing in Eastern China: preliminary findings. Arch Gerontol Geriatr. 2013 Jan-Feb;56(1):38-43.

34 Ng TP, Feng L, Lim WS, Chong MS, Lee TS, Yap KB, et al. Montreal Cognitive Assessment for screening mild cognitive impairment: variations in test performance and scores by education in Singapore. Dement Geriatr Cogn Disord. 2015;39(3-4):176-85.

35 Chen KL, Xu Y, Chu AQ, Ding D, Liang XN, Nasreddine ZS, et al. Validation of the Chinese version of Montreal Cognitive Assessment basic for screening mild cognitive impairment. J Am Geriatr Soc. 2016 Dec;64(12):e28590.

36 Mellor D, Lewis M, McCabe M, Byrne L, Wang T, Wang J, et al. Determining appropriate screening tools and cut-points for cognitive impairment in an elderly Chinese sample. Psychol Assess. 2016 Nov;28(11):1345-53.

37 Tan JP, Li N, Gao J, Wang LN, Zhao YM, Yu BC, et al. Optimal cutoff scores for dementia and mild cognitive impairment of the Montreal Cognitive Assessment among elderly and oldest-old Chinese population. J Alzheimers Dis. 2015;43(4):1403-12.

38 Delgado C, Araneda A, Behrens MI. Validación del instrumento Montreal Cognitive Assessment en español en adultos mayores de 60 años. Neurologia. DOI: 10.1016/j.nrl.2017.01.013.

39 Matías-Guiu JA, Fernández-Bobadilla R, Fernández-Oliveira A, Valles-Salgado M, Rognoni T, Cortés-Martínez A, et al. Normative Data for the Spanish Version of the Addenbrooke's Cognitive Examination III. Dement Geriatr Cogn Disord. 2016;41(5-6):243-50.

40 Gómez F, Zunzunegui M, Lord C, Alvarado B, García A. Applicability of the MoCA-S test in populations with little education in Colombia. Int J Geriatr Psychiatry. 2013 Aug;28(8):813-20. 